Ján Borsík, Mathematical Institute of Slovak Academy of Sciences,

Grešákova 6, 04001 Košice, Slovakia. e-mail:borsik@mail.saske.sk

Jozef Doboš, Department of Mathematics, Technical University, Letná 9, 04001 Košice, Slovakia, e-mail:dobos@ccsun.tuke.sk

Miroslav Repický, Mathematical Institute of Slovak Academy of Sciences, Jesenná 5, 04154 Košice, Slovakia e-mail:repicky@kosice.upjs.sk

\title{
SUMS OF QUASICONTINUOUS FUNCTIONS WITH CLOSED GRAPHS*
}

\begin{abstract}
We prove that every real-valued $\mathcal{B}_{1}^{*}$ function $f$ defined on a separable metric space $X$ is the sum of three quasicontinuous functions with closed graphs, and there is a $\mathcal{B}_{1}^{*}$ function which is not the sum of two quasicontinuous functions with closed graphs. Consequently, if $X$ is a separable metric space which is a Baire space in the strong sense, then the next three properties are equivalent: (1) $f$ is a $\mathcal{B}_{1}^{*}$ function, (2) $f$ is the sum of (at least) three quasicontinuous functions with closed graphs, and (3) $f$ is a piecewise continuous function.
\end{abstract}

\section{Introduction}

Let $X$ be a topological space. A function $f: X \rightarrow \mathbb{R}$ is said to be quasicontinuous (cliquish) at a point $x \in X$ if for every neighborhood $U$ of $x$ and every $\varepsilon>0$ there is an open set $G \subseteq U$ such that $|f(x)-f(y)|<\varepsilon$ for each $y \in G(|f(y)-f(z)|<\varepsilon$ for each $y, z \in G)$. A function $f$ is quasicontinuous (cliquish) if it is such at each point. A function $f: X \rightarrow \mathbb{R}$ has closed graph if the set $\{(x, f(x)): x \in X\}$ is a closed subset of $X \times \mathbb{R}$. A function $f: X \rightarrow \mathbb{R}$ is piecewise continuous if there are closed sets $X_{n} \subseteq X, n \in \mathbb{N}$ such that $X=\bigcup_{n=0}^{\infty} X_{n}$ and the restriction $f\left\lceil X_{n}\right.$ is continuous for each $n \in \mathbb{N}$. A function $f: X \rightarrow \mathbb{R}$ is a function of the class $\mathcal{B}_{1}^{*}$ (Baire-one-star function)

Key Words: Quasicontinuous function, piecewise continuous function, function of the class $\mathcal{B}_{1}^{*}$, function with closed graph

Mathematical Reviews subject classification: Primary 54C08; Secondary 54C30

Received by the editors June 2, 1999

*The authors were supported by VEGA, grants 2/5124/98, 1/5124/98, and 2/4034/97, respectively. 
if for every closed set $F \subseteq X$ there exists an open set $G \subseteq X$ such that $F \cap G \neq \emptyset$ and $f \uparrow(F \cap G)$ is continuous ([5]).

If $\mathcal{F} \subseteq \mathbb{R}^{X}$ is a family of real functions, then we denote by $\mathcal{G}(\mathcal{F})$ the group generated by $\mathcal{F}$. Further, denote by $\mathcal{Q}, \mathcal{U}, \mathcal{B}_{1}^{*}$, and $\mathcal{P}$ the families of all quasicontinuous functions, closed graph functions, Baire-one-star functions, and piecewise continuous functions (in $\mathbb{R}^{X}$ ), respectively.

Evidently, the sum of two quasicontinuous functions with closed graph need not be such. In this paper we will characterize the group generated by real quasicontinuous functions with closed graph. More precisely, we shall show that

$$
\mathcal{G}(\mathcal{Q U})=\mathcal{B}_{1}^{*}=\mathcal{P}=\mathcal{Q U}+\mathcal{Q U}+\mathcal{Q U}
$$

for separable metric spaces which are Baire spaces in the strong sense, in particular, for complete separable metric spaces. Further, we shall show that $\mathcal{Q U}+\mathcal{Q U} \neq \mathcal{B}_{1}^{*}$ (in spite of the facts that every $\mathcal{B}_{1}^{*}$ function on a metric space is the sum of two functions with closed graphs, [3], and that every cliquish function, and thus also every $\mathcal{B}_{1}^{*}$ function, is the sum of two quasicontinuous functions, [1]).

Recall that $X$ is a Baire space in the strong sense (or totally nonmeager) if every nonempty closed subspace of $X$ is a Baire space ([4]).

We use the following notation in the paper. Let $X$ be a metric space with a metric function $d: X \times X \rightarrow[0, \infty)$. For $x \in X, A, B \subseteq X$ and $\varepsilon>0$ we define

$$
\begin{aligned}
\operatorname{diam}(A) & =\sup \{d(a, b): a, b \in A\}, \\
\operatorname{dist}(x, A) & =\inf \{d(x, a): a \in A\}, \\
\operatorname{dist}(A, B) & =\inf \{d(a, b): a \in A \& b \in B\}, \\
S(x, \varepsilon) & =\{y \in X: d(x, y)<\varepsilon\}, \\
S(A, \varepsilon) & =\{y \in X: \operatorname{dist}(A, y)<\varepsilon\} .
\end{aligned}
$$

For a subset $A$ of $X, \mathrm{Cl}(A)$ and $\operatorname{Int}(A)$ denote the closure and the interior of $A$, respectively. The letters $\mathbb{N}, \mathbb{Q}$, and $\mathbb{R}$ stand for the set of natural, rational, and real numbers, respectively. For a function $f: X \rightarrow \mathbb{R}, D(f)$ denotes the set of all discontinuity points of $f$. The quantifier $\forall^{\infty} n$ abbreviates the quantifiers $(\exists m)(\forall n>m)$.

\section{A Characterization of $\mathcal{G}(\mathcal{Q U})$}

Lemma 2.1. Let $X$ be a topological space and let $f: X \rightarrow \mathbb{R}$ be a function. The following implications hold: 
(1) If $X$ is a Baire space, and $f$ is piecewise continuous, then $D(f)$ is nowhere dense.

(2) If $f$ is a $\mathcal{B}_{1}^{*}$ function, then $D(f)$ is nowhere dense.

(3) If $f$ is a quasicontinuous function with closed graph, then $D(f)$ is nowhere dense.

(4) $f$ is a $\mathcal{B}_{1}^{*}$ function if and only if $D(f\lceil F)$ is nowhere dense in $F$ for every closed set $F$ in $X$.

Proof. To obtain a contradiction (in the proofs of conditions (1)-(3)) let us assume that there is an open set $G \neq \emptyset$ such that $D(f)$ is dense in $G$.

(1) Let $X=\bigcup_{n=0}^{\infty} X_{n}$ where $X_{n}$ is closed and $f\left\lceil X_{n}\right.$ is continuous for each $n \in \mathbb{N}$. Since $G=\bigcup_{n=0}^{\infty} G \cap X_{n}$ is not meager, there is $m \in \mathbb{N}$ such that the set $G \cap X_{m}$ has nonempty interior $H$. Then $f$ is continuous at every point $x \in H$ which is a contradiction because $H \cap D(f) \neq \emptyset$.

(2) Let $F=\mathrm{Cl}(G)$. Since $f$ is a $\mathcal{B}_{1}^{*}$ function, there is an open set $H \subseteq X$ such that $F \cap H \neq \emptyset$ and $f \uparrow(F \cap H)$ is continuous. Then also $G \cap H \neq \emptyset$ and $(G \cap H) \cap D(f)=\emptyset$ which is a contradiction.

(3) Let us fix $x \in G$. Since $f$ is quasicontinuous, there is an open set $H \subseteq G$ such that $|f(x)-f(y)|<1$ for each $y \in H$. Then $f \nmid H$ is bounded and has closed graph (in $H \times \mathbb{R}$ ). Therefore $f \nmid H$ is continuous and $f$ is continuous at every point $x \in H$ which contradicts the choice of the set $G$.

(4) If $f$ is a $\mathcal{B}_{1}^{*}$ function and $F$ is closed in $X$, then $f \nmid F$ is a $\mathcal{B}_{1}^{*}$ function in $F$ and by condition (2) $D(f\lceil F)$ is nowhere dense in $F$. Conversely, if $f$ is not a $\mathcal{B}_{1}^{*}$ function, then by the definition there exists a closed set $F$ such that $D(f\lceil F)$ is dense in $F$.

Condition (3) in the previous lemma can be easily proved for cliquish functions with closed graphs.

A natural question is what is the relation between these three generalized continuity properties of functions. We can make several simple observations.

Lemma 2.2. If $X$ is a topological space and $f: X \rightarrow \mathbb{R}$ has closed graph, then $f$ is piecewise continuous.

Proof. The inverse images of compact subsets of $\mathbb{R}$ are closed subsets of $X$ and hence it is enough to take $X_{n}=f^{-1}([-n, n])$.

In [5] it is shown that the inclusion $\mathcal{B}_{1}^{*} \subseteq \mathcal{P}$ holds for metric spaces and the inclusion $\mathcal{P} \subseteq \mathcal{B}_{1}^{*}$ holds for all complete metric spaces. Next we will see that the equality holds for Baire metric spaces in the strong sense while this is not true for all Baire metric spaces. 
Lemma 2.3. If $X$ is a Baire space in the strong sense, then every piecewise continuous function $f: X \rightarrow \mathbb{R}$ is a $\mathcal{B}_{1}^{*}$ function.

Proof. Let $X=\bigcup_{n=0}^{\infty} X_{n}$ where $X_{n}$ is closed and $f\left\lceil X_{n}\right.$ is continuous for each $n \in \mathbb{N}$. Let $F \subseteq X$ be a closed set. Then $F=\bigcup_{n=0}^{\infty}\left(F \cap X_{n}\right)$ and since $F$ is a Baire space there is an open set $G$ in $X$ and $n \in \mathbb{N}$ such that $\emptyset \neq F \cap G \subseteq X_{n}$ and $f \uparrow(F \cap G)$ is continuous.

Remark 2.4. In spite of Lemma 2.1(3) and Lemma 2.3, it is not true that in a Baire space every quasicontinuous function with closed graph is a $\mathcal{B}_{1}^{*}$ function.

Proof. Let $\mathbb{Q}=\left\{r_{n}: n \in \mathbb{N}\right\}$ and let $A=\left\{x_{n, m}: n, m \in \mathbb{N}\right\}$ be a sequence of distinct irrational numbers. We define a metric $d$ on $X=\mathbb{Q} \cup A$ by

$$
\begin{aligned}
d(x, y) & =|x-y| \text { for } x, y \in \mathbb{Q} \\
d\left(x_{n^{\prime}, m}, r_{n}\right) & =1 /(m+1)+\left|r_{n^{\prime}}-r_{n}\right| \text { and } \\
d\left(x_{n_{1}, m_{1}}, x_{n_{2}, m_{2}}\right) & =1 /\left(m_{1}+1\right)+1 /\left(m_{2}+1\right)+\left|r_{n_{1}}-r_{n_{2}}\right| .
\end{aligned}
$$

Notice that $\left\{x_{n, m}\right\}$ is an open set in $X, A$ is a discrete open dense subset of $X$, and $X$ is a Baire space. We define $f: X \rightarrow \mathbb{R}$ by $f\left(r_{n}\right)=f\left(x_{n, m}\right)=n$. Then $f$ is a quasicontinuous function with closed graph and $f$ is not a $\mathcal{B}_{1}^{*}$ function because $\mathbb{Q}$ is closed in $X$ and $f \uparrow(\mathbb{Q} \cap G)$ is not continuous for any open set $G$ in $X$ such that $\mathbb{Q} \cap G \neq \emptyset$.

The aim of the paper is the proof of the next theorem.

Theorem 2.5. Let $X$ be a separable metric space which is a Baire space in the strong sense and let $f: X \rightarrow \mathbb{R}$. The following conditions are equivalent:

(1) $f$ is the sum of three quasicontinuous functions with closed graphs.

(2) $f$ is the sum of at least three quasicontinuous functions with closed graphs.

(3) $f$ is piecewise continuous.

(4) $f$ is of the class $\mathcal{B}_{1}^{*}$.

Proof. The implication $(1) \rightarrow(2)$ is trivial, the implication $(2) \rightarrow(3)$ is a consequence of Lemma 2.2, and the equality $\mathcal{P}+\mathcal{P}=\mathcal{P}$. The implication $(3) \rightarrow(4)$ is Lemma 2.3, and the implication $(4) \rightarrow(1)$ is Theorem 4.1 .

Remark 2.6. The assumption $X$ is a Baire space in the strong sense can be neither removed nor replaced by the assumption that $X$ is a Baire space, see Remark 2.4. 
Remark 2.7. The separability assumption is necessary for the proof of the implication (4) $\rightarrow(1)$, namely for the proof of Lemma 3.3 .

Remark 2.8. The sum of three functions cannot be replaced by the sum of two functions in condition (1) of Theorem 2.5. Namely, the function $f$ defined by $f(x)=1$, if $x=0$, and $f(x)=0$, if $x \neq 0$, is a $\mathcal{B}_{1}^{*}$ function, but it is not the sum of two quasicontinuous functions with closed graphs.

Proof. Assume that $f=f_{1}+f_{2}$ where $f_{1}, f_{2}$ are quasicontinuous functions with closed graphs. Then $f_{1}(x)=-f_{2}(x)$ for every $x \neq 0$. As $f_{1}$ is quasicontinuous at 0 , there exists a sequence $\left\{x_{n}\right\}_{n=0}^{\infty}$ convergent to 0 such that $\lim _{n \rightarrow \infty} f_{1}\left(x_{n}\right)=f_{1}(0)$ and then $\lim _{n \rightarrow \infty} f_{2}\left(x_{n}\right)=-f_{1}(0)$. Then, as $f_{2}$ has closed graph, $f_{2}(0)=-f_{1}(0)$ and we have this contradiction: $1=f(0)=$ $f_{1}(0)+f_{2}(0)=0$.

Problem 2.9. Characterize the family $\mathcal{Q U}+\mathcal{Q U}$.

We will need the next easy property of quasicontinuous functions.

Lemma 2.10. If $f: X \rightarrow \mathbb{R}, K$ is an open set, and $x \in \mathrm{Cl}(K)$ is such that $f \uparrow \mathrm{Cl}(K)$ is quasicontinuous at $x$, then $f$ is quasicontinuous at $x$.

\section{Systems of Closed Nowhere Dense Sets}

Lemma 3.1 ([1], Lemma 3.1). Let $X$ be a metric space, $F \subseteq X$ be a closed nowhere dense set and let $G \subseteq X$ be an open set such that $F \subseteq \mathrm{Cl}(G)$. Then there is a family $\mathcal{K}=\bigcup_{n=0}^{\infty} \mathcal{K}_{n}$ of nonempty open subsets of $X$ such that the following conditions hold:

(i) The set $E_{n}=\bigcup\left\{\mathrm{Cl}(K): K \in \mathcal{K}_{n}\right\}$ is closed and $E_{n} \subseteq S(F, 2 / n) \cap G \backslash F$ for every $n \in \mathbb{N}$.

(ii) $(\forall x \in X \backslash F)(\exists V$ a neighburhood of $x)|\{K \in \mathcal{K}: V \cap \mathrm{Cl}(K) \neq \emptyset\}| \leq 1$.

(iii) $(\forall x \in F)(\forall V$ a neighborhood of $x)\left(\forall^{\infty} n\right)\left(\exists K \in \mathcal{K}_{n}\right) \mathrm{Cl}(K) \subseteq V$.

In particular, $\mathrm{Cl}\left(\bigcup_{n=0}^{\infty} E_{n}\right)=\bigcup_{n=0}^{\infty} E_{n} \cup F$.

Proof. The construction is by induction on $n \in \mathbb{N}$. Assume that $\mathcal{K}_{i}, i<n$ have been constructed. Put

$$
\begin{aligned}
T_{n} & =G \cap S(F, 1 / n) \backslash\left(F \cup \bigcup_{i<n} E_{i}\right), \\
\alpha_{n}(x) & =\frac{1}{4} \cdot \operatorname{dist}\left(x, F \cup(X \backslash G) \cup \bigcup_{i<n} E_{i}\right) .
\end{aligned}
$$

Let $S_{n} \subseteq T_{n}$ be a maximal set with the property that $d(x, y)>1 / n$ for $x \neq y$ in $S_{n}$. We set $\mathcal{K}_{n}=\left\{S\left(x, \alpha_{n}(x)\right): x \in S_{n}\right\}$. 
The more detailed proof of the above lemma can be found in [1] and the same arguments we use in the proof of Lemma 3.3 below. We need this result for the next consequence.

Lemma 3.2. Let $H_{0}$ be a nowhere dense subset of a metric space $X$ and let $\mathcal{H}$ be a countable family of closed subsets of $H_{0}$ linearly ordered by inclusion $\subseteq$. Then there exists an order isomorphism $\varphi$ from $\mathcal{H}$ onto a family of open subsets of $X \backslash H_{0}$ such that $H_{0} \cap \mathrm{Cl}(\varphi(H))=H$ for every $H \in \mathcal{H}$.

Proof. Without loss of generality we can assume that $\emptyset, H_{0} \in \mathcal{H}$. Let $\mathfrak{n}$ be the cardinality of $\mathcal{H}$; i.e., either $\mathfrak{n}$ is an integer or $\mathfrak{n}=\omega$, and let $\left\{H_{n}: n<\mathfrak{n}\right\}$ be an enumeration of $\mathcal{H}$ such that $H_{1}=\emptyset$ (and $H_{0}$ is the given nowhere dense set). We define $\varphi\left(H_{n}\right)$ by induction for $n<\mathfrak{n}$. Set $\varphi\left(H_{0}\right)=X \backslash H_{0}, \varphi\left(H_{1}\right)=\emptyset$ and let us assume that $n>1$ and the open sets $\varphi\left(H_{i}\right)$ are defined for $i<n$. There are $j, k<n$ such that $H_{j} \subseteq H_{n} \subseteq H_{k}$ and for every $i<n$ either $H_{i} \subseteq H_{j}$ or $H_{k} \subseteq H_{i}$. Set $F=H_{n}$ and $G=\varphi\left(H_{k}\right)$ in Lemma 3.1 and let $\mathcal{K}$ be the obtained system of open sets. Let $V=\bigcup \mathcal{K}$. Then $V \subseteq \varphi\left(H_{k}\right)$ and $\operatorname{Cl}(V)=$ $\bigcup_{i=0}^{\infty} E_{i} \cup H_{n}$ while $\bigcup_{i=0}^{\infty} E_{i} \subseteq \varphi\left(H_{k}\right) \subseteq X \backslash H_{0}$. Therefore $H_{0} \cap \mathrm{Cl}(V)=H_{n}$. Finally, $\mathrm{Cl}\left(V \cup \varphi\left(H_{j}\right)\right)=\mathrm{Cl}(V) \cup \mathrm{Cl}\left(\varphi\left(H_{j}\right)\right)$ and $H_{0} \cap \mathrm{Cl}\left(\varphi\left(H_{j}\right)\right)=H_{j} \subseteq$ $H_{n}$. Therefore we can set $\varphi\left(H_{n}\right)=V \cup \varphi\left(H_{j}\right)$.

Lemma 3.3. Let $X$ be a metric space and let $\xi$ be a countable ordinal number. Let $\left\{F_{\alpha}\right\}_{\alpha \leq \xi}$ be a sequence of closed nowhere dense sets such that $F_{\xi}=\emptyset$ and $F_{\beta} \varsubsetneqq F_{\alpha}$ for $\alpha<\beta \leq \xi$. There exists a system $\mathcal{L}=\bigcup_{\alpha<\xi} \mathcal{L}^{\alpha}$ of disjoint nonempty open sets, where $\mathcal{L}^{\alpha}=\bigcup_{n=0}^{\infty} \mathcal{L}_{n}^{\alpha}$ is a disjoint union, such that

(1) $(\forall K \in \mathcal{L}) \mathrm{Cl}(K) \cap F_{0}=\emptyset$.

(2) $\left(\forall x \in X \backslash F_{0}\right)(\exists V$ a neighbourhood of $x)|\{K \in \mathcal{L}: V \cap \mathrm{Cl}(K) \neq \emptyset\}| \leq 1$.

(3) $\left(\forall x \in F_{0} \backslash F_{\alpha}\right)(\exists V$ a neighbourhood of $x)\left(\forall K \in \bigcup_{\beta \geq \alpha} \mathcal{L}^{\beta}\right) V \cap \mathrm{Cl}(K)=$ $\emptyset$.

(4) $\left(\forall x \in F_{\alpha}\right)(\forall V$ a neighbourhood of $x)\left(\forall^{\infty} n\right)\left(\exists K \in \mathcal{L}_{n}^{\alpha}\right) \mathrm{Cl}(K) \subseteq V$.

(5) $\left(\forall x \in F_{0}\right)(\forall K \in \mathcal{L}) \mathrm{Cl}(K) \subseteq S(x, 2 \operatorname{dist}(x, \mathrm{Cl}(K)))$.

Proof. By Lemma 3.2 let us fix a descending sequence of open sets $\left\{V_{\alpha}\right\}_{\alpha \leq \xi}$ such that $V_{\alpha} \cap F_{0}=\emptyset$ and $F_{\alpha}=F_{0} \cap \mathrm{Cl}\left(V_{\alpha}\right)$. Let $(\lambda, \rho): \omega \rightarrow \xi \times \omega$ be a bijection such that

$$
n \leq m \text { and } \lambda(n)=\lambda(m) \text { implies } \rho(n) \leq \rho(m) .
$$

By induction we define the families $\mathcal{L}_{\rho(n)}^{\lambda(n)}$ so that the following conditions hold:

(i) $\operatorname{diam}(K)<1 /(2 n)$ for $K \in \mathcal{L}_{\rho(n)}^{\lambda(n)}$. 
(ii) The set $E_{n}=\bigcup\left\{\mathrm{Cl}(K): K \in \mathcal{L}_{\rho(n)}^{\lambda(n)}\right\}$ is closed and even every set $E_{n}(\mathcal{A})=\bigcup\{\mathrm{Cl}(K): K \in \mathcal{A}\}$ is closed for $\mathcal{A} \subseteq \mathcal{L}_{\rho(n)}^{\lambda(n)}$.

(iii) $E_{n} \subseteq S\left(F_{\lambda(n)}, 2 / n\right) \cap V_{\lambda(n)}$.

(iv) $\left(\forall x \in F_{\lambda(n)}\right)\left(\exists K \in \mathcal{L}_{\rho(n)}^{\lambda(n)}\right) K \subseteq S(x, 2 / n)$.

(v) $\left(\forall x \in F_{0}\right)\left(\forall K \in \mathcal{L}_{\rho(n)}^{\lambda(n)}\right) \mathrm{Cl}(K) \subseteq S(x, 2 \operatorname{dist}(x, \mathrm{Cl}(K)))$.

Assume that the families $\mathcal{L}_{\rho(i)}^{\lambda(i)}, i<n$ have been constructed. Put

$$
\begin{aligned}
T_{n} & =S\left(F_{\lambda(n)}, 1 / n\right) \cap V_{\lambda(n)} \backslash\left(F_{0} \cup \bigcup_{i<n} E_{i}\right), \\
\alpha_{n}(x) & =\frac{1}{4} \cdot \operatorname{dist}\left(x, F_{0} \cup\left(X \backslash V_{\lambda(n)}\right) \cup \bigcup_{i<n} E_{i}\right) .
\end{aligned}
$$

Let $S_{n} \subseteq T_{n}$ be a maximal set with the property that $d(x, y)>1 / n$ for $x \neq y$ in $S_{n}$. We set

$$
\mathcal{L}_{\rho(n)}^{\lambda(n)}=\left\{S\left(x, \alpha_{n}(x)\right): x \in S_{n}\right\} .
$$

Now we verify conditions (i) $-(\mathrm{v})$.

(i) For $x \in S_{n}$ we have $\operatorname{dist}\left(x, F_{\lambda(n)}\right)<1 / n$ because $x \in T_{n}$, and hence $\alpha_{n}(x)<1 /(4 n)$. Therefore $\operatorname{diam}\left(S\left(x, \alpha_{n}(x)\right)\right)<1 /(2 n)$.

(ii) For any $x \neq y$ in $S_{n}, d(x, y)>1 / n$ and $\alpha_{n}(x), \alpha_{n}(y)<1 /(4 n)$. Therefore $\operatorname{dist}\left(S\left(x, \alpha_{n}(x)\right), S\left(y, \alpha_{n}(y)\right)\right) \geq d(x, y)-\alpha_{n}(x)-\alpha_{n}(y)>1 /(2 n)$.

(iii) By definition of $\alpha_{n}(x)$ we can see that $\mathrm{Cl}\left(S\left(x, \alpha_{n}(x)\right)\right) \subseteq V_{\lambda(n)}$ for $x \in S_{n}$. Moreover, since $x \in S\left(F_{\lambda(n)}, 1 / n\right)$,

$$
\mathrm{Cl}\left(S\left(x, \alpha_{n}(x)\right)\right) \subseteq S\left(F_{\lambda(n)}, 1 / n+\alpha_{n}(x)\right) \subseteq S\left(F_{\lambda(n)}, 2 / n\right) .
$$

(iv) Let $x \in F_{\lambda(n)}$. Since $F_{\lambda(n)}$ is nowhere dense and disjoint from $\bigcup_{i<n} E_{i}$, there is $y \in S(x, 1 /(2 n)) \backslash\left(F_{\lambda(n)} \cup \bigcup_{i<n} E_{i}\right)$. Notice that

$$
S_{n} \cap S(x, 1 /(2 n)+1 / n) \neq \emptyset
$$

since otherwise $y$ could be added to $S_{n}$ contradicting the maximality of $S_{n}$. Now, for $y \in S_{n} \cap S(x, 1 /(2 n)+1 / n)$ we have

$$
S\left(y, \alpha_{n}(y)\right) \subseteq S\left(x, 1 /(2 n)+1 / n+\alpha_{n}(y)\right) \subseteq S(x, 2 / n) .
$$

(v) Let $x \in F_{0}$ and $K \in \mathcal{L}_{\rho(n)}^{\lambda(n)}, K=S\left(y, \alpha_{n}(y)\right)$. Then

$$
\alpha_{n}(y) \leq \operatorname{dist}\left(y, F_{0}\right) / 4 \leq d(y, x) / 4 .
$$

Therefore $\operatorname{dist}(x, K) \geq d(y, x)-\alpha_{n}(y)>2 \alpha_{n}(y)$ and

$$
K \subseteq S\left(x, \operatorname{dist}(x, K)+2 \alpha_{n}(y)\right) \subseteq S(x, 2 \operatorname{dist}(x, K)) .
$$


Now we show that conditions (1)-(5) are satisfied. Conditions (1), (4) and (5) are consequences of conditions (iii), (iv) and (v), respectively.

(2) Let $x \in X \backslash F_{0}$, $\operatorname{dist}\left(x, F_{0}\right)>4 / k$ for some $k$. If $n \geq k$, then by (iii) $E_{n} \subseteq S\left(F_{\lambda(n)}, 2 / n\right) \subseteq S\left(F_{0}, 2 / k\right)$. It follows that $S(x, 2 / k) \cap \bigcup_{n \geq k} E_{n}=$ $\emptyset$. Now there are two possibilities: Either, $x \in \mathrm{Cl}\left(K_{0}\right)$ for some $K_{0} \in \mathcal{L}$ and then the set $V=S(x, 2 / k) \backslash \bigcup_{n<k}\left(E_{n} \backslash \mathrm{Cl}\left(K_{0}\right)\right)$ is open, $x \in V$, and $V \cap K \neq \emptyset$ if and only if $K=K_{0}$ for $K \in \mathcal{L}$. Or, $x \notin \bigcup_{n<k} E_{n}$, and then $V=S(x, 2 / k) \backslash \bigcup_{n<k} E_{n}$ is open, $x \in V$, and $V \cap K=\emptyset$ for every $K \in \mathcal{L}$.

(3) Let $\alpha \leq \beta \leq \xi$ and let $x \in F_{0} \backslash F_{\alpha}$. Then, by the choice of the set $V_{\alpha}$, $x \in X \backslash \mathrm{Cl}\left(V_{\alpha}\right) \subseteq X \backslash \mathrm{Cl}\left(V_{\beta}\right)$, and by (iii), $\mathrm{Cl}(K) \subseteq V_{\beta}$ for every $K \in \mathcal{L}^{\beta}$.

\section{Main Result}

Theorem 4.1. Let $X$ be a separable metric space. Then every $\mathcal{B}_{1}^{*}$ function $f: X \rightarrow \mathbb{R}$ is the sum of three quasicontinuous functions with closed graphs.

Remark 4.2. As we already mentioned every $\mathcal{B}_{1}^{*}$ function is piecewise continuous. However in Theorem $4.1 \mathcal{B}_{1}^{*}$ can't be replaced by piecewise continuous because for $X=\mathbb{Q}$ every function is piecewise continuous while there exists a function which is not cliquish and hence not every function is a sum of quasicontinuous functions.

Proof. Let $f \in \mathcal{B}_{1}^{*}$. Let us introduce the following notation:

$$
f^{+}=\max \{f, 0\}, \quad f^{-}=\min \{f, 0\},
$$

and for a closed set $A \subseteq X$ let $h_{A}: X \backslash A \rightarrow \mathbb{R}$ be defined by

$$
h_{A}(x)=1 / \operatorname{dist}(x, A) \text {, if } A \neq \emptyset \text {, and } h_{A}(x)=0 \text {, if } A=\emptyset .
$$

By induction we define the following sequence of closed nowhere dense subsets of $X$ :

$$
\begin{aligned}
F_{0} & =\operatorname{Cl}(D(f)), \\
F_{\alpha+1} & =\operatorname{Cl}\left(D\left(f\left\lceil F_{\alpha}\right)\right),\right. \\
F_{\alpha} & =\bigcap_{\beta<\alpha} F_{\beta} \text { for } \alpha \text { a limit ordinal. }
\end{aligned}
$$

By Lemma 2.1(4) the set $F_{0}$ is nowhere dense and $F_{\alpha+1}$ is nowhere dense in $F_{\alpha}$. As $X$ is separable every descending sequence of closed sets in $X$ must be countable. Let $\xi<\omega_{1}$ be the least ordinal for which $F_{\xi}=\emptyset$. If $\xi=0$, then $f$ is continuous and $f=f+0+0$ is the sum of three continuous functions. 
Therefore let us assume that $\xi>0$. By Lemma 3.3 there exists a system $\mathcal{L}=\bigcup_{\alpha<\xi} \mathcal{L}^{\alpha}$ of disjoint nonempty open sets satisfying conditions (1)-(5). For $\alpha<\xi$ and $K \in \mathcal{L}^{\alpha}$ let us fix $b_{K} \in K$ and $a_{K} \in F_{\alpha} \backslash F_{\alpha+1}$ such that $0<$ $d\left(b_{K}, a_{K}\right)<2 \operatorname{dist}\left(b_{K}, F_{\alpha} \backslash F_{\alpha+1}\right)$. This is possible because $\operatorname{Cl}(K) \cap F_{0}=\emptyset$. By condition (2) of Lemma 3.3 the set $D=F_{0} \cup \bigcup\{\mathrm{Cl}(K): K \in \mathcal{L}\}$ is closed. We define quasicontinuous functions $f_{1}, f_{2}, f_{3}: X \rightarrow \mathbb{R}$ with closed graphs as follows:

For $x \in F_{\alpha} \backslash F_{\alpha+1}, \alpha<\xi$,

$$
\begin{aligned}
& f_{1}(x)=f^{+}(x)+h_{F_{\alpha+1}}(x), \\
& f_{2}(x)=h_{F_{\alpha+1}}(x), \\
& f_{3}(x)=f^{-}(x)-2 h_{F_{\alpha+1}}(x) .
\end{aligned}
$$

For $x \in \mathrm{Cl}(K), K \in \mathcal{L}_{3 n}^{\alpha}, n \in \mathbb{N}, \alpha<\xi$,

$$
\begin{aligned}
& f_{1}(x)=f^{+}\left(a_{K}\right)+h_{F_{\alpha+1}}\left(a_{K}\right) \\
& f_{2}(x)=f^{+}(x)+h_{F_{\alpha}}(x) \\
& f_{3}(x)=f^{-}(x)-h_{F_{\alpha}}(x)-f^{+}\left(a_{K}\right)-h_{F_{\alpha+1}}\left(a_{K}\right) .
\end{aligned}
$$

For $x \in \mathrm{Cl}(K), K \in \mathcal{L}_{3 n+1}^{\alpha}, n \in \mathbb{N}, \alpha<\xi$,

$$
\begin{aligned}
& f_{1}(x)=f^{+}(x)+h_{F_{\alpha}}(x), \\
& f_{2}(x)=h_{F_{\alpha+1}}\left(a_{K}\right), \\
& f_{3}(x)=f^{-}(x)-h_{F_{\alpha}}(x)-h_{F_{\alpha+1}}\left(a_{K}\right) .
\end{aligned}
$$

For $x \in \mathrm{Cl}(K), K \in \mathcal{L}_{3 n+2}^{\alpha}, n \in \mathbb{N}, \alpha<\xi$,

$$
\begin{aligned}
& f_{1}(x)=f^{+}(x)+h_{F_{\alpha}}(x)-f^{-}\left(a_{K}\right)+2 h_{F_{\alpha+1}}\left(a_{K}\right), \\
& f_{2}(x)=f^{-}(x)-h_{F_{\alpha}}(x), \\
& f_{3}(x)=f^{-}\left(a_{K}\right)-2 h_{F_{\alpha+1}}\left(a_{K}\right) .
\end{aligned}
$$

For $x \in X \backslash D$,

$$
\begin{aligned}
& f_{1}(x)=f^{+}(x)+h_{D}(x), \\
& f_{2}(x)=h_{D}(x), \\
& f_{3}(x)=f^{-}(x)-2 h_{D}(x) .
\end{aligned}
$$

Easily we can verify that $f=f_{1}+f_{2}+f_{3}$. We prove that the functions $f_{1}$, $f_{2}, f_{3}$ are quasicontinuous and have closed graphs.

The functions $f$ and $h_{F_{\alpha}}$ are continuous on the open set $X \backslash F_{0}$ and hence the functions $f^{+}, f^{-}, h_{F_{\alpha}}$ are continuous at every point of $x \in X \backslash D$ and at 
every point $x \in \mathrm{Cl}(K)$ for $K \in \mathcal{L}, \alpha<\xi$. Further, the function $h_{D}$ is continuous on the open set $X \backslash D$. The functions $f_{1}, f_{2}, f_{3}$ are constructed from these functions in such a way that the restrictions $f_{i} \uparrow \mathrm{Cl}(K)$ and $f_{i} \uparrow(X \backslash D)$ for $K \in \mathcal{L}$ and $i=1,2,3$ are continuous. Since $X \backslash F_{0}=(X \backslash D) \cup \bigcup_{K \in \mathcal{L}} \mathrm{Cl}(K)$, by Lemma 2.10 it follows that the functions $f_{1}, f_{2}, f_{3}$, are quasicontinuous at every $x \in X \backslash F_{0}$.

Let $x \in F_{0}$, i.e., $x \in F_{\alpha} \backslash F_{\alpha+1}$ for some $\alpha<\xi$. Let $U$ be a neighbourhood of $x$ and let $\varepsilon>0$ be arbitrary. Since the functions $f\left\lceil F_{\alpha}\right.$ (and also $f^{+} \uparrow F_{\alpha}$, $\left.f^{-} \mid F_{\alpha}\right)$ and $h_{F_{\alpha+1}} \uparrow F_{\alpha}$ are continuous at $x$, there exists $V \subseteq U$, a neighborhood of $x$, such that

$$
\max \left\{\left|f^{+}(x)-f^{+}(y)\right|,\left|f^{-}(x)-f^{-}(y)\right|,\left|h_{F_{\alpha+1}}(x)-h_{F_{\alpha+1}}(y)\right|\right\}<\varepsilon / 3
$$

for each $y \in V \cap F_{\alpha}$. Let $\delta>0$ be such that $S(x, 3 \delta) \subseteq V$. By condition (4) of Lemma 3.3 there exists $n \in \mathbb{N}$ and $K_{1} \in \mathcal{L}_{3 n}^{\alpha}, K_{2} \in \mathcal{L}_{3 n+1}^{\alpha}, K_{3} \in \mathcal{L}_{3 n+2}^{\alpha}$ such that $\mathrm{Cl}\left(K_{i}\right) \subseteq S(x, \delta)$ for $i=1,2,3$. Then $\operatorname{dist}\left(b_{K_{i}}, F_{\alpha} \backslash F_{\alpha+1}\right)<\delta$ and hence $d\left(b_{K_{i}}, a_{K_{i}}\right)<2 \delta$. It follows that $a_{K_{i}} \in V \cap F_{\alpha}$ because $d\left(x, a_{K_{i}}\right) \leq$ $d\left(x, b_{K_{i}}\right)+d\left(b_{K_{i}}, a_{K_{i}}\right)<\delta+2 \delta=3 \delta$. Now applying the second case in the definition of $f_{1}$, the third case in the definition of $f_{2}$, and the fourth case in the definition of $f_{3}$ we obtain

$$
\begin{aligned}
& \left|f_{1}(x)-f_{1}(y)\right| \leq\left|f^{+}(x)-f^{+}\left(a_{K_{1}}\right)\right|+\left|h_{F_{\alpha+1}}(x)-h_{F_{\alpha+1}}\left(a_{K_{1}}\right)\right| \text { for } y \in K_{1}, \\
& \left|f_{2}(x)-f_{2}(y)\right| \leq\left|h_{F_{\alpha+1}}(x)-h_{F_{\alpha+1}}\left(a_{K_{2}}\right)\right| \text { for } y \in K_{2}, \\
& \left|f_{3}(x)-f_{3}(y)\right| \leq\left|f^{-}(x)-f^{-}\left(a_{K_{3}}\right)\right|+2\left|h_{F_{\alpha+1}}(x)-h_{F_{\alpha+1}}\left(a_{K_{3}}\right)\right| \text { for } y \in K_{3},
\end{aligned}
$$

and since $a_{K_{i}} \in V \cap F_{\alpha}$, using the inequality (*) we get $\left|f_{i}(x)-f_{i}(y)\right|<\varepsilon$ for all $y \in K_{i}$ and $i=1,2,3$. Therefore, the functions $f_{1}, f_{2}, f_{3}$ are quasicontinuous also at every $x \in F_{0}$ and we have proved that these functions are quasicontinuous everywhere.

It remains to prove that graphs of functions $f_{1}, f_{2}, f_{3}$ are closed subsets of $X \times \mathbb{R}$. It is enough to prove (see [2]) that for every $x \in X$ and $i=1,2,3$

$$
C\left(f_{i}, x\right)=\bigcap\{\mathrm{Cl}(f(U)): U \text { is a neighbourhood of } x\}=\left\{f_{i}(x)\right\} .
$$

For $x \in X \backslash D, C\left(f_{i}, x\right)=\left\{f_{i}(x)\right\}$ because $f_{i}$ 's are continuous on $X \backslash D$. For every $x \in D$ we find a sequence $V_{m}, m \in \mathbb{N}$ of neighborhoods of $x$ such that

$$
f_{i}(y) \in(-\infty,-m) \cup\left(f_{i}(x)-1 / m, f_{i}(x)+1 / m\right) \cup(m, \infty) .
$$

for each $m \in \mathbb{N}, y \in V_{m}$ and $i=1,2,3$. This will end the proof since

$$
\bigcap_{m=0}^{\infty} \mathrm{Cl}\left(f_{i}\left(V_{m}\right)\right)=\left\{f_{i}(x)\right\}=C\left(f_{i}, x\right), \text { for } i=1,2,3 .
$$


Let $x \in D$. There are two cases:

Case 1. $x \in \mathrm{Cl}(K)$ for some $K \in \mathcal{L}_{n}^{\alpha}$ with $\alpha<\xi$ and $n \in \mathbb{N}$. By condition (2) of Lemma 3.3 there exists a neighborhood $V$ of $x$ such that $\mathrm{Cl}(L) \cap V=\emptyset$ for every $L \in \mathcal{L} \backslash\{K\}$. As $f_{i} \uparrow \mathrm{Cl}(K)$ are continuous, we can find neighborhoods $W_{m}$ of $x$ for $m \in \mathbb{N}$ such that $\left|f_{i}(x)-f_{i}(y)\right|<1 / m$ for $y \in W_{m} \cap \mathrm{Cl}(K)$ and $i=1,2$, 3. Let $r \in \mathbb{N}$ be such that $\operatorname{dist}\left(x, F_{0}\right)>1 / r$. Then the sets $V_{m}=W_{m} \cap V \cap S(x, 1 /(r+m))$ for $m \in \mathbb{N}$ are neighborhoods of $x$ disjoint from $F_{0}$ and

$$
V_{m}=\left(V_{m} \cap \mathrm{Cl}(K)\right) \cup\left(V_{m} \backslash D\right) .
$$

For $y \in V_{m} \cap \mathrm{Cl}(K)$ we have $\left|f_{i}(x)-f_{i}(y)\right|<1 / m$. For $y \in V_{m} \backslash D$ we have $\operatorname{dist}(y, D) \leq \operatorname{dist}(y, x)<1 /(r+m)$ because $x \in D$ and hence $h_{D}(y)>m$. Since $f^{+}(y) \geq 0, f^{-}(y) \leq 0$, by the fifth cases of the definitions of functions $f_{1}, f_{2}, f_{3}$ we get $f_{1}(y)>m, f_{2}(y)>m$, and $f_{3}(y)<-2 m$.

Case 2. $x \in F_{0}$ and hence $x \in F_{\alpha} \backslash F_{\alpha+1}$ for some $\alpha<\xi$. The functions $f \nmid F_{\alpha}$ and $h_{F_{\alpha+1}}$ are continuous at $x$; so we can find neighborhoods $W_{m}$ of $x$ such that

$$
\max \left\{\left|f^{+}(x)-f^{+}(y)\right|,\left|f^{-}(x)-f^{-}(y)\right|,\left|h_{F_{\alpha+1}}(x)-h_{F_{\alpha+1}}(y)\right|\right\}<1 /(3 m)
$$

for each $y \in W_{m} \cap F_{\alpha}$ and $m \in \mathbb{N}$. Let $0<\delta_{m}<1 /(6 m)$ be such that $S\left(x, 6 \delta_{m}\right) \subseteq W_{m}$. By condition (3) of Lemma 3.3 there exists an open neighborhood $V$ of $x$ such that $V \cap \mathrm{Cl}(K)=\emptyset$ for $K \in \bigcup_{\beta>\alpha} \mathcal{L}^{\beta}$. The sets $V_{m}=V \cap S\left(x, \delta_{m}\right) \backslash F_{\alpha+1}$ for $m \in \mathbb{N}$ are neighborhoods of $x$ and $V_{m}=$ $\left(V_{m} \backslash D\right) \cup\left(V_{m} \cap F_{\alpha} \backslash F_{\alpha+1}\right) \cup\left(V_{m} \cap F_{0} \backslash F_{\alpha}\right) \cup\left(V_{m} \cap \bigcup_{\beta \leq \alpha} \bigcup_{K \in \mathcal{L}^{\beta}} \mathrm{Cl}(K)\right)$. So for
$y \in V_{m}$ we have four subcases:

Case 2a. $y \in V_{m} \backslash D$. Then $h_{D}(y)>m$, because $x \in D$ and $d(x, y)<1 / m$, and hence $f_{1}(y)>m, f_{2}(y)>m$, and $f_{3}(y)<-2 m$.

Case 2b. $y \in V_{m} \cap\left(F_{\alpha} \backslash F_{\alpha+1}\right)$. Then by the choice of $W_{m}$

$$
\begin{aligned}
& \left|f_{1}(x)-f_{1}(y)\right| \leq\left|f^{+}(x)-f^{+}(y)\right|+\left|h_{F_{\alpha+1}}(x)-h_{F_{\alpha+1}}(y)\right|<1 / m, \\
& \left|f_{2}(x)-f_{2}(y)\right|=\left|h_{F_{\alpha+1}}(x)-h_{F_{\alpha+1}}(y)\right|<1 / m, \\
& \left|f_{3}(x)-f_{3}(y)\right| \leq\left|f^{-}(x)-f^{-}(y)\right|+2\left|h_{F_{\alpha+1}}(x)-h_{F_{\alpha+1}}(y)\right|<1 / m .
\end{aligned}
$$

Case 2c. $y \in V_{m} \cap\left(F_{0} \backslash F_{\alpha}\right)$. There is $\beta<\alpha$ such that $y \in F_{\beta} \backslash F_{\beta+1}$. As $x \in F_{\alpha} \subseteq F_{\beta+1}$, we have $\operatorname{dist}\left(y, F_{\beta+1}\right) \leq d(x, y)<1 / m$ and $h_{F_{\beta+1}}(y)>m$. Therefore $f_{1}(y)>m, f_{2}(y)>m$, and $f_{3}(y)<-2 m$. 
Case 2d. $y \in V_{m} \cap \mathrm{Cl}(K)$ for some $K \in \mathcal{L}_{3 n+j}^{\beta}, j \in\{0,1,2\}, n \in \mathbb{N}$, and $\beta \leq \alpha$. As $x \in F_{\alpha} \subseteq F_{\beta}$, $\operatorname{dist}\left(y, F_{\beta}\right) \leq d(y, x) \leq \delta_{m}<1 / m$. Therefore $h_{F_{\beta}}(y)>m$ and hence

$$
\begin{array}{ll}
f_{1}(y)>m, \text { if } j=1,2, & f_{2}(y)>m, \text { if } j=0, \\
& f_{2}(y)<-m, \text { if } j=2,
\end{array}
$$

As $\operatorname{dist}(x, \mathrm{Cl}(K)) \leq d(x, y) \leq \delta_{m}$ and $\mathrm{Cl}(K) \subseteq S(x, 2 \operatorname{dist}(x, \mathrm{Cl}(K))) \subseteq$ $S\left(x, 2 \delta_{m}\right)$ by condition (5) of Lemma 3.3 , we have

$$
d\left(x, a_{K}\right) \leq d\left(x, b_{K}\right)+d\left(b_{K}, a_{K}\right)<2 \delta_{m}+4 \delta_{m}=6 \delta_{m} .
$$

So if $\beta=\alpha, a_{K} \in W_{m} \cap F_{\alpha}$ and by (4.3),

if $j=0$, then

$$
\left|f_{1}(x)-f_{1}(y)\right| \leq\left|f^{+}(x)-f^{+}\left(a_{K}\right)\right|+\left|h_{F_{\alpha+1}}(x)-h_{F_{\alpha+1}}\left(a_{K}\right)\right|<1 / m,
$$

if $j=1$, then

$$
\left|f_{2}(x)-f_{2}(y)\right|=\left|h_{F_{\alpha+1}}(x)-h_{F_{\alpha+1}}\left(a_{K}\right)\right|<1 / m,
$$

and if $j=2$, then

$$
\left|f_{3}(x)-f_{3}(y)\right| \leq\left|f^{-}(x)-f^{-}\left(a_{K}\right)\right|+2\left|h_{F_{\alpha+1}}(x)-h_{F_{\alpha+1}}\left(a_{K}\right)\right|<1 / m .
$$

If $\beta<\alpha$, then $x \in F_{\alpha} \subseteq F_{\beta+1}$ and hence $\operatorname{dist}\left(a_{K}, F_{\beta+1}\right) \leq d\left(a_{K}, x\right)<6 \delta_{m}<$ $1 / m$. Then $h_{F_{\beta+1}}\left(a_{K}\right)>m$ and hence

$$
f_{1}(y)>m, \text { if } j=0, \quad f_{2}(y)>m, \text { if } j=1, \quad f_{3}(y)<-2 m, \text { if } j=2 .
$$

In all cases we have proved the property (4.2) and so the proof of Theorem 4.1 is complete.

\section{References}

[1] J. Borsík, Sums of quasicontinuous functions defined on pseudometrizable spaces, Real Anal. Exchange 22 (1996/97), 328-337.

[2] J. Borsík, Local characterizations of functions with closed graphs, Demonstratio Math. 29 (1996), 643-650.

[3] J. Doboš, Sums of closed graph functions, Tatra Mt. Math. Publ. 14 (1998), 9-11.

[4] R. C. Haworth and R. A. McCoy, Baire Spaces, Dissertationes Math. (Rozprawy Mat.) 141, Polish Acad. Sci., Warsaw 1977.

[5] B. Kirchheim, Baire one star functions, Real Anal. Exchange 18 (1992/93), 385-399. 